\title{
Correction to: A quantitative imaging biomarker for predicting disease-free-survival-associated histologic subgroups in lung adenocarcinoma
}

\author{
Lin $\mathrm{Lu}^{1} \cdot$ Deling Wang ${ }^{2} \cdot$ Lili Wang ${ }^{3} \cdot$ Linning $\mathrm{E}^{4} \cdot$ Pingzhen Guo ${ }^{1} \cdot{\mathrm{Zhiming} \mathrm{Li}^{5} \cdot \text { Jin Xiang }}^{6} \cdot \mathrm{Hao} \mathrm{Yang}^{1} \cdot \mathrm{Hui}^{\mathrm{Li}}{ }^{2}$. \\ Shaohan Yin ${ }^{2} \cdot$ Lawrence H. Schwartz ${ }^{1}$. Chuanmiao Xie ${ }^{2} \cdot$ Binsheng Zhao ${ }^{1}$
}

Published online: 22 July 2020

(C) European Society of Radiology 2020

\section{Correction to: European Radiology \\ https://doi.org/10.1007/s00330-020-06663-6}

The original version of this article, published on 21 February 2020 , unfortunately contained a mistake. The optimal Intensity-Skewness cutoff of the imaging biomarker is $<-1.5$. Therefore, in the abstract, the last sentence of section "Results" should read: "A criterion of Intensity-Skewness $<-1.5$, which indicated high tumor density, showed high specificity of $96 \%$ (sensitivity $46 \%$ ) and $99 \%$ (sensitivity $53 \%$ ) on predicting the mid/poor DFS group in the training and external validation cohorts, respectively."

The second key point should read: "An Intensity-Skewness of $<-1.5$ has high specificity in predicting the mid/poor disease-free survival histologic patient group in both the training cohort and the external validation cohort."

And in "Results," the last sentence of paragraph "Cutoff analysis" should read: "As shown in Fig. 4, the cutoff Intensity-Skewness <- 1.5 could achieve high specificity to predict the mid/poor DFS group, especially in the training and external validation cohorts which had specificity of $96 \%$ (sensitivity 53\%) and 99\% (sensitivity 46\%), respectively."

Publisher's note Springer Nature remains neutral with regard to jurisdictional claims in published maps and institutional affiliations.
The online version of the original article can be found at https://doi.org/ 10.1007/s00330-020-06663-6

\footnotetext{
Chuanmiao Xie

xchuanm@sysucc.org.cn

Binsheng Zhao

bz2166@cumc.columbia.edu

1 Department of Radiology, Columbia University Medical Center, 710 West 168th Street, B26, New York, NY 10032, USA
}

2 Department of Radiology, State Key Laboratory of Oncology in South China, Collaborative Innovation Center for Cancer Medicine, Sun Yat-sen University Cancer Center, Guangzhou 510060, People's Republic of China
3 Department of Molecular Pathology, The Affiliated Hospital of Qingdao University, Qingdao University, Wutaishan Road 1677, Qingdao 266000, Shandong, People's Republic of China

4 Department of Radiology, Shanxi Bethune Hospital, 99 Longcheng Street, Taiyuan 030032, Shanxi, People's Republic of China

5 Department of Radiology, The Affiliated Hospital of Qingdao University, Qingdao University, Wutaishan Road 1677, Qingdao 266000, Shandong, People's Republic of China

6 Department of Pathology, State Key Laboratory of Oncology in South China, Collaborative Innovation Center for Cancer Medicine, Sun Yat-sen University Cancer Center, Guangzhou 510060, People's Republic of China 